\title{
Optimal design of a microgripper-type actuator based on AIN/Si heterogeneous bimorph
}

Ruiz, D. P.; Diaz-Molina, A.; Sigmund, Ole; Donoso, Alberto; Bellido, J.C.; Sanchez-Rojas, J. L.

Published in:

Proceedings of SPIE

Link to article, DOI:

$10.1117 / 12.2266498$

Publication date:

2017

Document Version

Publisher's PDF, also known as Version of record

Link back to DTU Orbit

Citation (APA):

Ruiz, D. P., Diaz-Molina, A., Sigmund, O., Donoso, A., Bellido, J. C., \& Sanchez-Rojas, J. L. (2017). Optimal design of a microgripper-type actuator based on AIN/Si heterogeneous bimorph. In Proceedings of SPIE (Vol. 10246). [1024606] SPIE - International Society for Optical Engineering. Proceedings of SPIE - The International Society for Optical Engineering https://doi.org/10.1117/12.2266498

\section{General rights}

Copyright and moral rights for the publications made accessible in the public portal are retained by the authors and/or other copyright owners and it is a condition of accessing publications that users recognise and abide by the legal requirements associated with these rights.

- Users may download and print one copy of any publication from the public portal for the purpose of private study or research.

- You may not further distribute the material or use it for any profit-making activity or commercial gain

- You may freely distribute the URL identifying the publication in the public portal 


\section{Optimal design of a microgripper-type actuator based on AIN/Si heterogeneous bimorph}

D. Ruiz

A. Díaz-Molina

O. Sigmund

A. Donoso

J. C. Bellido

J. L. Sánchez-Rojas 


\title{
Optimal design of a microgripper-type actuator based on AlN/Si heterogeneous bimorph
}

\author{
Ruiz D. ${ }^{a}$, Díaz-Molina A. ${ }^{\mathrm{b}}$, Sigmund O. ${ }^{\mathrm{c}}$, Donoso A. ${ }^{\mathrm{d}}$, Bellido JC. ${ }^{\mathrm{e}}$, and Sánchez-Rojas JL. ${ }^{\mathrm{f}}$ \\ ${ }^{a, d, e}$ Department of Mathematics, ETSII, University of Castilla-la Mancha, Ciudad Real, Spain \\ b,f Microsystems, actuators and sensors group, ETSII, University of Castilla-la Mancha, Ciudad \\ Real, Spain \\ ${ }^{\mathrm{c}}$ Department of Mechanical Engineering, Technical University of Denmark, Lyngby, Denmark
}

\begin{abstract}
This work presents a systematic procedure to design piezoelectrically actuated microgrippers. Topology optimization combined with optimal design of electrodes is used to maximize the displacement at the output port of the gripper. The fabrication at the microscale leads us to overcome an important issue: the difficulty of placing a piezoelectric film on both top and bottom of the host layer. Due to the non-symmetric lamination of the structure, an out-of-plane bending spoils the behaviour of the gripper. Suppression of this out-of-plane deformation is the main novelty introduced. In addition, a robust formulation approach is used in order to control the length scale in the whole domain and to reduce sensitivity of the designs to small manufacturing errors.
\end{abstract}

Keywords: Topology optimization, Piezoelectric actuator, Simultaneous design, Electrode profile, Microtransducers, Robust formulation.

\section{INTRODUCTION}

Topology optimization is a conceptual tool whose use is not restricted to structural design: design of compliant mechanisms ${ }^{1,2}$, band gap ${ }^{3}$, metamaterial design ${ }^{4}$ and dynamics problems ${ }^{5}$ amongst other, are fields where its contribution has been crucial.

The design of piezoelectric devices is a field where topology optimization has been widely used in the last decade. This tool allows to improve the physical response of this kind of devices. In a pioneering work in this area ${ }^{6}$, the unit cell of structures is optimized for improving piezoelectric features in hydrophone applications. Concerning the design of piezoelectric actuators, in ${ }^{7}$ is presented a method to design piezoelectric in-plane actuators by optimizing the host structure, but keeping fixed the layer of piezoelectric material. The optimization of piezoelectric layers with a three-layer model, with two piezoelectric films symmetrically bonded to the host structure is treated in ${ }^{8}$. The optimizization at the same time of the host structure and the piezoelectric distribution is presented in ${ }^{9}$ and ${ }^{10}$. The inclusion of a third variable, the spatial distribution of the control voltage (related to the polarization of the piezoelectric layers) in the optimization problem, is addressed in ${ }^{11}$ and improved by introducing an interpolation scheme in the tri-level actuation voltage term in ${ }^{12}$. In ${ }^{13}$ and ${ }^{14}$ the authors present new results for in-plane and out-of-plane piezoelectric transducers, respectively.

Regarding simultaneous optimization of both, host structure and polarization profile of the piezoelectric film, in ${ }^{15}$ and ${ }^{16}$ is introduced a systematic method for optimizing transducers for statics and mode filtering, respectively. In these works the authors make the assumption of two piezoelectric films perfectly bonded to the top and bottom of the host structure. Either in-phase or out-of-phase polarization of the piezoelectric layers makes the structure move in-plane or out-of-plane, respectively. Unfortunately, fabrication at the microscale presents an important limitation: placing two piezoelectric layer symmetrically ${ }^{17}$, hence only one piezoelectric film can be deposited on the top of the host structure. When fabricating a sensor this is not truly a problem, since the deformation is produced by an external force. However, when the device is working as actuator it moves in the in-plane and out-of-plane directions.

Further author information: (Send correspondence to David Ruiz)

David Ruiz: E-mail: David.Ruiz@uclm.es

Smart Sensors, Actuators, and MEMS VIII, edited by Luis Fonseca, Mika Prunnila, Erwin Peiner, Proc. of SPIE Vol. 10246, 1024606 - (c) 2017 SPIE · CCC code: 0277-786X/17/\$18 · doi: 10.1117/12.2266498 
TOP VIEW

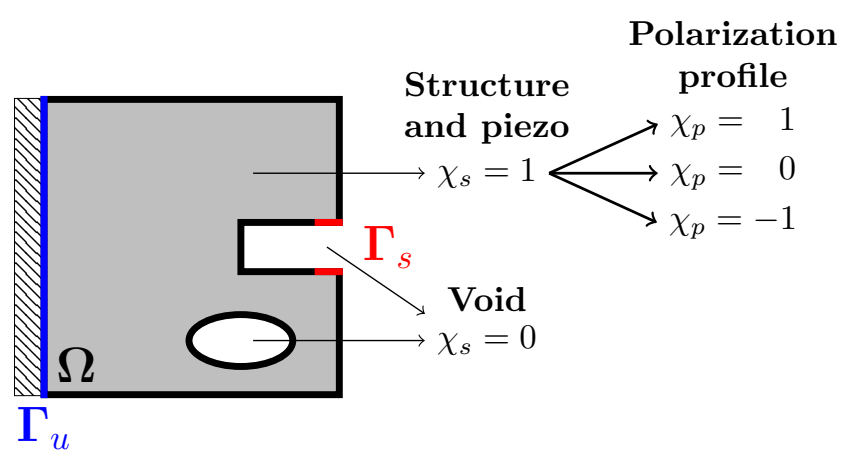

SIDE VIEW

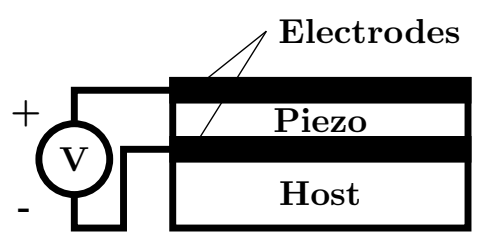

Figure 1. Top and side view of the piezoelectric device.

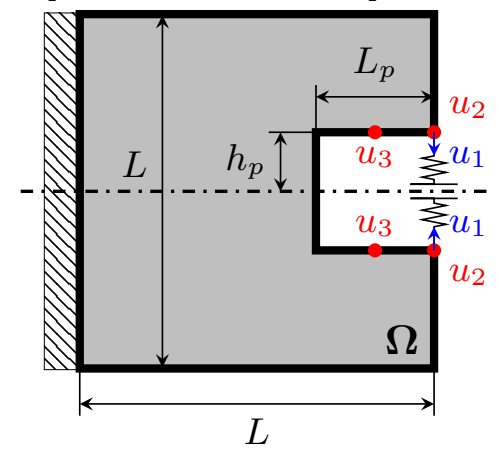

Figure 2. Design domain.

Motivated by this real difficulty, the objective of the present work is to design a piezoelectrically actuated microgripper including some constraints on the out-of-plane bending of the structure in some points of interest. Additionally, having manufacturability in mind, the so-called robust formulation ${ }^{18}$ is implemented in the model with two purposes: controlling the length scale of the designs in both solid and void regions, and minimizing the sensitivity of the target under fabrication errors.

The work is organized as follows. In Section 2 we present the discrete formulation of the problem. Section 3 is focused on the robust approach formulation. The numerical implementation of the problem is briefly described in Section 4. Section 5 is devoted to show the optimized designs. Finally, in Section 6 the conclusions of the work are presented.

\section{TOPOLOGY OPTIMIZATION OF PIEZOELECTRIC MICROGRIPPER}

We consider as a design domain $\Omega$ a rectangular plate clamped at its left side. On the top surface the host structure is perfectly bonded with a piezoelectric layer (which is sandwiched between two electrodes) of negligible stiffness compared to the plate as shown in figure 1. $\Gamma_{u}$ stands for the left clamped side of the boundary and $\Gamma_{s}$ stands for the end of the jaws, where the vertical displacement is suppressed.

The piezoelectric layer is polarized through the voltage applied to the electrodes. Thanks to the piezoelectric effect the electric field generates a mechanical stress over the piezoelectric material, which in turn deforms the host structure. The unsymmetrical configuration of the layers causes the out-of-plane bending of the structure, which disturbs the in-plane behaviour and needs to be suppressed. Figure 2 shows a passive area defined between the jaws of the gripper (white color) representing an empty space to grab the objects. The output of the gripper is modeled as a spring with a stiffness $k_{\text {out }}$ that depends on the application.

The objective is the maximization of the in-plane displacement $u_{1}$, while the out-of-plane bending in two points placed at the jaws is suppressed as much as possible. This vertical displacement is suppressed in two point to avoid the rotation of the jaws. 
The optimization problem involves two density variables, as shown in figure 1 . The first is the structural density $\rho_{s} \in[\mathbf{0}, \mathbf{1}]$, which represents the structure layout $\left(\rho_{s}=1\right)$ and void $\left(\rho_{s}=0\right)$. The second variable is the polarization profile $\rho_{p} \in[-1,1]$, meaning negative or positive polarity. The domain $\Omega$ is dicretized is $n_{e}$ finite elements, with two variables per element. The latter variable plays an important role in this problem since only the part of the host structure covered by electrode is electrically affected and then subjected to the piezoelectric force.

The formulation of the problem written as a topology optimization problem becomes:

$\max _{\boldsymbol{\rho}_{s}, \boldsymbol{\rho}_{p}}: u_{1}\left(\boldsymbol{\rho}_{s}, \boldsymbol{\rho}_{p}\right)$

s.t.: In-plane and out-of-plane equilibrium equation:

$$
\left\{\begin{array}{l}
\left(\mathbf{K}_{i p}\left(\boldsymbol{\rho}_{s}\right)+\mathbf{1}_{\text {out }} k_{\text {out }}\right) \mathbf{U}_{i p}=\mathbf{F}_{i p}\left(\boldsymbol{\rho}_{s}, \boldsymbol{\rho}_{p}\right) \\
\mathbf{K}_{o p}\left(\boldsymbol{\rho}_{s}\right) \mathbf{U}_{o p}=\mathbf{F}_{o p}\left(\boldsymbol{\rho}_{s}, \boldsymbol{\rho}_{p}\right) .
\end{array}\right.
$$

Displacements at the jaws:

$\left\{\begin{array}{l}u_{1}=\mathbf{L}_{1}^{T} \mathbf{U}_{i p} \\ u_{2}=\mathbf{L}_{2}^{T} \mathbf{U}_{o p} \\ u_{3}=\mathbf{L}_{3}^{T} \mathbf{U}_{o p} .\end{array}\right.$

Constraints:

$$
\left\{\begin{array}{l}
\left(\frac{u_{2}}{u_{1}}\right)^{2}-\varepsilon_{d}^{2} \leq 0 \\
\left(\frac{u_{3}}{u_{1}}\right)^{2}-\varepsilon_{d}^{2} \leq 0 \\
\frac{\mathbf{1}^{T} \boldsymbol{\rho}_{s}}{V}-1 \leq 0 \\
\boldsymbol{\rho}_{s} \in[\mathbf{0}, \mathbf{1}] \\
\boldsymbol{\rho}_{p} \in[-\mathbf{1}, \mathbf{1}],
\end{array}\right.
$$

where $u_{1}$ represents the in-plane displacement to be maximized and $u_{2}$ and $u_{3}$ the out-of-plane displacements to be suppressed. $\mathbf{L}_{1}, \mathbf{L}_{2}$ and $\mathbf{L}_{3}$ are zero vectors with 1 in the degree of freedom of interest. Subscripts $i p$ and $o p$ stand for the in-plane and out-of-plane cases, respectively. $\mathbf{K}$ is the stiffness matrix, $\mathbf{U}$ is the displacement vector and $\mathbf{F}$ is the piezoelectric force, for each motion case. $\mathbf{1}_{\text {out }}$ is a zero vector with 1 in the degree of freedom to be maximized and $k_{\text {out }}$ is the stiffness that models the output.

The original formulation of the problem involves integer variables that need to be relaxed into the density variables. The well-known SIMP approach (Solid Isotropic Material with Penalization ${ }^{19}$ and ${ }^{20}$ ) is used with this purpose. The Young's modulus of each element depends on the element density as follows:

$E_{e}=\left(\rho_{s e}\right)^{p}\left(E_{0}-E_{\text {min }}\right)+E_{\text {min }}$,

where $E_{0}$ is the Young's modulus of the base material, $E_{\min }>0$ is a small value used to avoid singularities in the stiffness matrix and $p$ is the penalization exponent (typically $p=3$ ).

Concerning the finite element model, for the in-plane case bilinear elements with 8 degrees of freedom are used, and for the out-of-plane, Kirchhoff plate elements with 12 degrees of freedom. The detailed computations for the stiffness matrices and force vectors can be found in ${ }^{21}$ and are not stated here. The expressions for the flexural and extensional components of the piezoelectric force can be found in ${ }^{22}$ and ${ }^{23}$. In addition, the same powerlaw dependence $\left(R_{e}=\rho_{s e}^{p}\right)$ used in the SIMP approach is used for the piezoelectric force generation. This interpolation scheme $\mathbf{R}$ premultiplies the piezoelectric force. This is a realistic way to model the force produced in void elements where there is no electrode. 


\section{ROBUST FORMULATION}

Manufacturing errors are very common in fabrication processes, specially at the microscale. These small errors can perturb the functionality of the gripper, so it would be desirable to minimize the sensitivity of the goal (the displacement at the output port) to under- and over-etching. A way to do that is by using the robust topology optimization approach presented in ${ }^{18}$. The method consists in the use of three different projections: eroded, intermediate and dilated, where the worst performing design is optimized. The projection used in this work is a generalization of the previous schemes used in ${ }^{24}$ and ${ }^{25}$ and is expressed as follows:

$\bar{\rho}_{s e}=\frac{\tanh \left(\beta_{0} \eta\right)+\tanh \left(\beta_{0}\left(\tilde{\rho}_{s e}-\eta\right)\right)}{\tanh \left(\beta_{0} \eta\right)+\tanh \left(\beta_{0}(1-\eta)\right)}$,

where $\beta_{0}$ is a tunable parameter that defines the smoothness of the projection and $\eta$ is the threshold, which can take values between 0 and 1 . The filtered densities $\tilde{\rho}_{s e}$ are projected to 1 if their values are bigger than the threshold and to 0 if not. The filtered densities $\tilde{\rho}_{s e}\left({ }^{24}\right.$ and $\left.{ }^{26}\right)$ are expressed as follows:

$\tilde{\rho}_{s e}=\frac{\sum_{j \in N_{e}} w\left(\mathbf{x}_{j}\right) v_{j} \rho_{s j}}{\sum_{j \in N_{e}} w\left(\mathbf{x}_{j}\right) v_{j}}$,

where $\mathbf{x}$ is the center of the element $j, v_{j}$ is the volume of the $j$-th element, $N_{e}$ is the neightbourhood of element $e$ within a certain filter radius $r$ specified by $N_{e}=\left\{j \mid\left\|\mathbf{x}_{j}-\mathbf{x}_{e}\right\| \leq r\right\}$, and $w\left(\mathbf{x}_{j}\right)=r-\left\|\mathbf{x}_{j}-\mathbf{x}_{e}\right\|$.

From now on the super index $q$ stands for the projection, $e$ for erode, $i$ for intermediate and $d$ por dilate.

The optimization problem formulated in terms of the robust approach may be expressed as:

$\max _{\boldsymbol{\rho}_{s}, \boldsymbol{\rho}_{p}} \min : \quad\left\{u_{1}^{e}\left(\overline{\boldsymbol{\rho}}_{s}^{e}\left(\boldsymbol{\rho}_{s}\right), \boldsymbol{\rho}_{p}\right), u_{1}^{i}\left(\overline{\boldsymbol{\rho}}_{s}^{i}\left(\boldsymbol{\rho}_{s}\right), \boldsymbol{\rho}_{p}\right), u_{1}^{d}\left(\overline{\boldsymbol{\rho}}_{s}^{d}\left(\boldsymbol{\rho}_{s}\right), \boldsymbol{\rho}_{p}\right)\right\}$,

s.t.: In-plane and out-of-plane equilibrium equations:

$\left\{\begin{array}{l}\left(\mathbf{K}_{i p}^{q}\left(\overline{\boldsymbol{\rho}}_{s}^{q}\right)+\mathbf{1}_{\text {out }} k_{\text {out }}\right) \mathbf{U}_{i p}^{q}=\mathbf{F}_{i p}^{q}\left(\overline{\boldsymbol{\rho}}_{s}^{q}, \boldsymbol{\rho}_{p}\right), \quad q \equiv e, i, d \\ \mathbf{K}_{o p}^{q}\left(\overline{\boldsymbol{\rho}}_{s}^{q}\right) \mathbf{U}_{o p}^{q}=\mathbf{F}_{o p}^{q}\left(\overline{\boldsymbol{\rho}}_{s}^{q}, \boldsymbol{\rho}_{p}\right), \quad q \equiv e, i, d .\end{array}\right.$

Displacements at the jaws:

$\begin{cases}u_{1}^{q}=\mathbf{L}_{1}^{T} \mathbf{U}_{i p}^{q}, & q \equiv e, i, d \\ u_{2}^{q}=\mathbf{L}_{2}^{T} \mathbf{U}_{o p}^{q}, & q \equiv e, i, d \\ u_{3}^{q}=\mathbf{L}_{3}^{T} \mathbf{U}_{o p}^{q}, & q \equiv e, i, d .\end{cases}$

Constraints:

$\left\{\begin{array}{l}\left(\frac{u_{2}^{q}}{u_{1}^{q}}\right)^{2}-\varepsilon_{d}^{2} \leq 0, \quad q \equiv e, i, d \\ \left(\frac{u_{3}^{q}}{u_{1}^{q}}\right)^{2}-\varepsilon_{d}^{2} \leq 0, \quad q \equiv e, i, d \\ \frac{\mathbf{1}^{T} \overline{\boldsymbol{\rho}}_{s}^{d}}{V_{d}^{*}}-1 \leq 0 \quad q \equiv e, i, d \\ \boldsymbol{\rho}_{s} \in[\mathbf{0}, \mathbf{1}] \\ \boldsymbol{\rho}_{p} \in[-\mathbf{1}, \mathbf{1}],\end{array}\right.$

where $V_{d}^{*}$ is the maximum volume constraint computed over the dilated design. The expression for this volume is:

$V_{d}^{*}=\frac{V^{*}}{V_{i}} V_{d}$ 
with $V_{i}$ and $V_{d}$ being the volume for the intermediate and the dilated designs and $V^{*}$ the maximum volume prescribed for the intermediate design. This value is updated during the optimization process.

The objective max - min function proposed in the problem is not differentiable. In order to alleviate this issue, the optimization problem is reformulated by using the so-called bound formulation. We replace the objective function (1) by:

$\max : \quad \beta$

$\boldsymbol{\rho}_{s}, \boldsymbol{\rho}$

and we add a new constraint to (2):

$\beta-u_{1}^{q} \leq 0, \quad q \equiv e, i, d$,

where $\beta$ is an additional bound variable that does not depend on the design variables $\boldsymbol{\rho}_{s}$ and $\boldsymbol{\rho}_{p}$ and circumvent non-differentiability issue with the max-min function.

\section{NUMERICAL APPROACH}

In this section the numerical implementation of the problem is presented. A gradient-based method, the MMA (Method of Moving Asymptotes ${ }^{27}$ ), is used to solve the optimization problem. This method requires information of the objective function, the constraints and the sensitivities of both, but these computations are straightforward for this particular problem and that is the reason why they are not included here.

The complete algorithm process looks like:

1. Choose the dimension of the plate and the properties of the materials that will be used. Boundary conditions and the parameters $\varepsilon_{d}$ and $k_{\text {out }}$ must be fixed.

2. Initialize the design variables $\boldsymbol{\rho}_{s}$ and $\boldsymbol{\rho}_{p}$.

3. Compute the physical densities $\overline{\boldsymbol{\rho}}_{s}$ by filtering the initial structural density $\boldsymbol{\rho}_{s}$ and then projecting with three different thresholds.

4. Solve the 6 finite element problems (3 in-plane and 3 out-of-plane).

5. Extract the displacements $u_{1}^{q}, u_{2}^{q}$ and $u_{3}^{q}$ and compute the constraints.

6. Compute the sensitivities of the objective function and the constraints.

7. Update design variables by using MMA.

8. Check the stop criteria, until convergence update the value of $\beta_{0}$ and $V_{d}^{*}$ and go back to step 3 .

At this point it is important to remark that thanks to the symmetry of the problem only half of the domain needs to be simulated and optimized.

\section{EXAMPLES}

In this section we illustrate our approach with several examples. The software used to solve this problem is programmed with Matlab and afterwards the physical behaviour is checked with CoventorWare.

The material of the host structure is silicon, and the one of the piezoelectric layer is AlN. The Young's modulus of the silicon and the piezoelectric layers are $130 \mathrm{GPa}$ and $345 \mathrm{GPa}$, respectively. This last value is only used to compute the piezoelectric force, while this stiffness is neglected when computing the global stiffness matrix, since its thickness is small compared to the thickness of the plate. The stiffness of the void area is set to $130 \mathrm{~Pa}$ (negligible compared to the silicon Young's modulus) in order to avoid singularities in the stiffness matrix. The Poisson's ratio is set to 0.28 . The thickness of the layers are $10 \mu \mathrm{m}$ for the silicon and $1 \mu \mathrm{m}$ for the AlN, respectively. The piezoelectric constant of AlN used to model the piezoelectric force is $d_{31}=2 \times 10^{-12} \mathrm{~m} / \mathrm{V}$. 

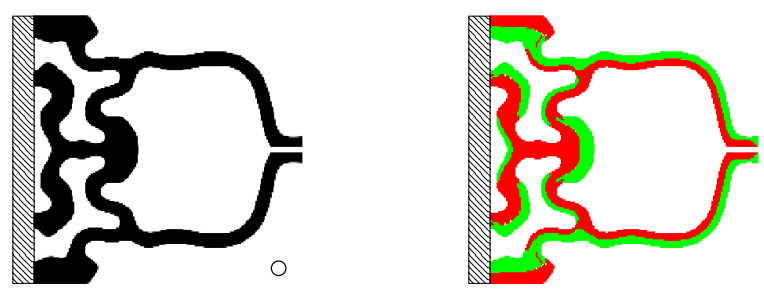

(a) Intermediate projection
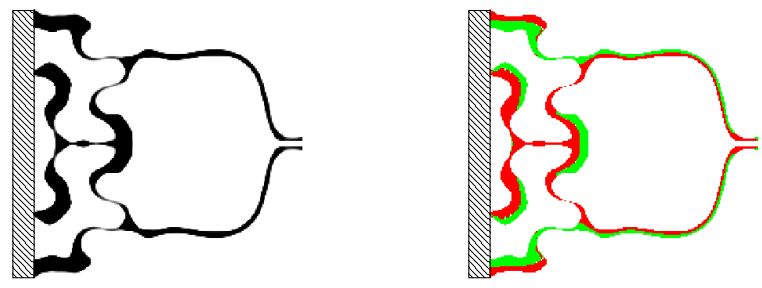

(b) Erode projection
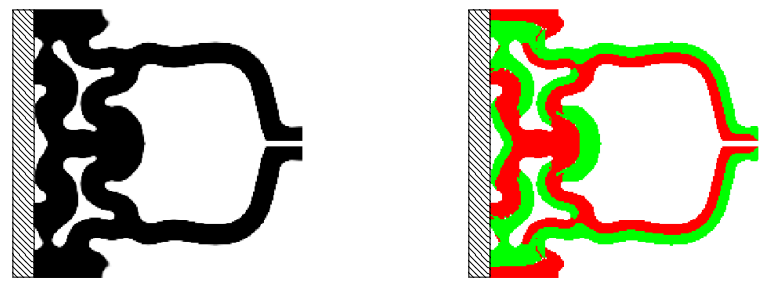

(c) Dilate projection

Figure 3. Optimized microgripper for $k_{\text {out }}=20 \mathrm{~N} / \mathrm{m}$ and $L=5000 \mu \mathrm{m}$. The circle in the blue print design represents the minimum length scale.

The input voltage is set to $10 \mathrm{~V}$. The coefficient $\varepsilon_{d}$, which relates the optimized displacement and the suppressed one, is fixed to $1 \%$. Concerning the geometrical constants, the size of the passive area is set to $L_{p}=1000 \mu \mathrm{m}$ and $h_{p}=50 \mu \mathrm{m}$. The points where the out-of-plane bending is canceled are separated $25 \mu \mathrm{m}$. The threshold of the projection is $\eta=0.3$, and the smoothness starts at $\beta=1$ and is doubled every 50 iterations up to $\beta=32$. All the parameters presented above keep constant in all the examples. The rest of them, such as the size of the domain or the number of finite elements, will change and will be introduced before every example.

Figure 3 shows the first microgripper designed. In this example the length of the domain is set to $L=5000 \mu \mathrm{m}$ and it is discretized with a mesh of $200 \times 200$ finite elements. The stiffness of the spring is set to $k_{\text {out }}=20 \mathrm{~N} / \mathrm{m}$ and the feature size to $250 \mu \mathrm{m}$. The structure variable $\overline{\boldsymbol{\rho}}_{s}$ is represented in figure 3(left), where black and white mean solid and void, respectively. Note that thanks to the projection method there are no gray areas (that represent microstructure). Figure 3(right) shows the polarization profile, meaning green and red positive and negative polarity, respectively. It is remarkable that the whole structure is covered with electrode, since null polarity areas are not electrically affected. The design obtained with the intermediate projection is called blue print design. This is the one where the length scale is controlled in both void and solid, and then the one that is fabricated. The output displacement obtained at the output port is $u_{1}^{i}=2.46 \mu \mathrm{m}$ and the suppressed out-of-plane displacements $u_{2}^{i}$ and $u_{3}^{i}$ are smaller than the $1 \%$ of the former.

Figure 4 shows the 3D modeling of figure 6(a) with the FEM software. The color represents the out-of-plane bending of the gripper. Although lower than the in-plane displacement, the vertical displacement near the jaws is not negligible, but clearly demonstrates the potential of our model to limit the bending of actuators fabricated 

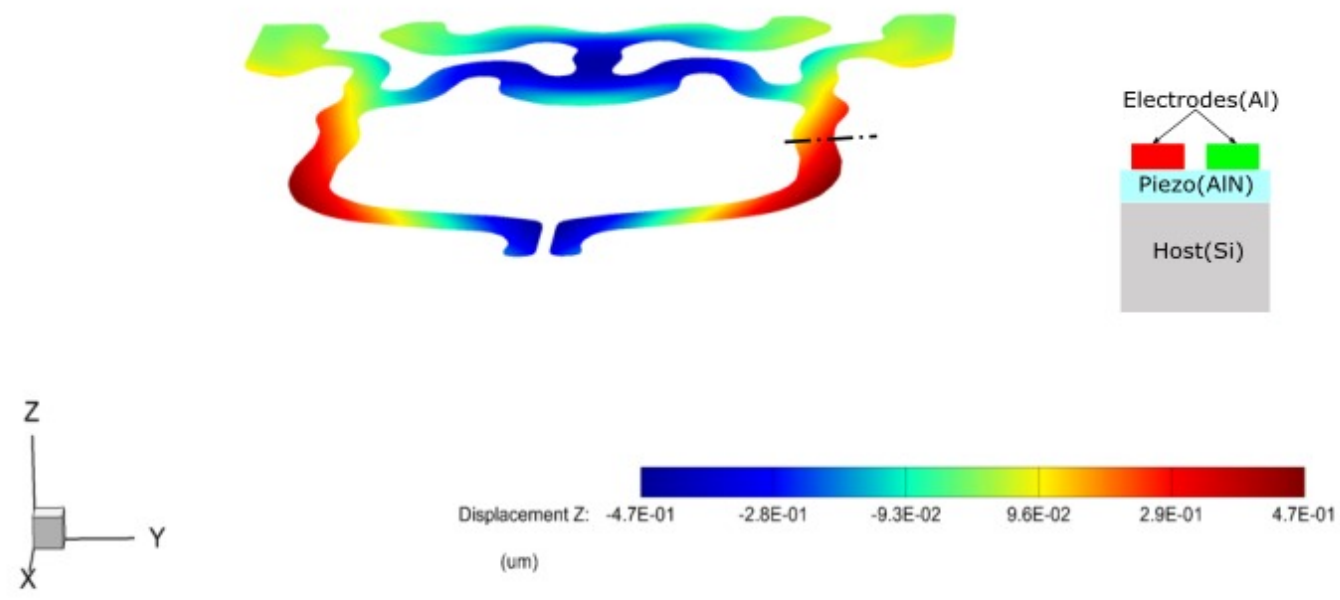

(a) Undeformed gripper
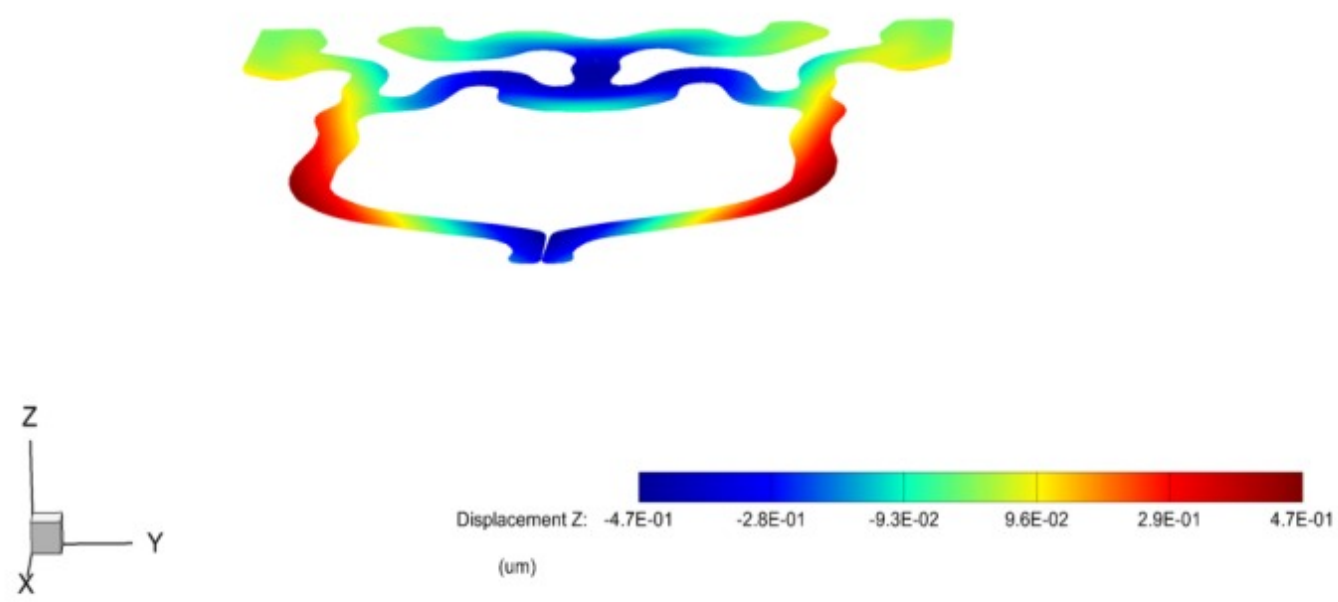

(b) Deformed gripper

Figure 4. 3D modeling with the FEM software. Colors showing the out-of-plane displacement. The inset corresponds to a cross section along the dotted line, remarking the spacing between electrodes. 

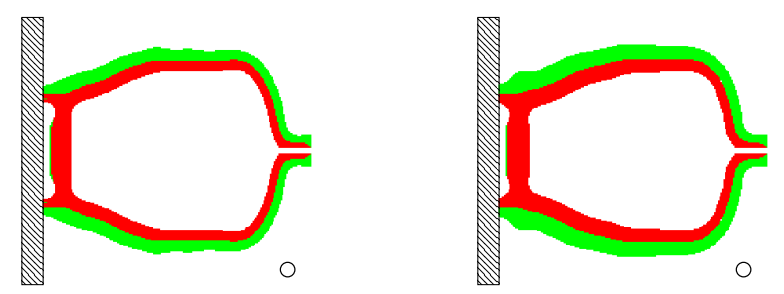

Figure 5. Polarization profile for $L=5000 \mu \mathrm{m}$ and $k_{\text {out }}=100 \mathrm{~N} / \mathrm{m}$ (left) and $k_{\text {out }}=200 \mathrm{~N} / \mathrm{m}$ (right).
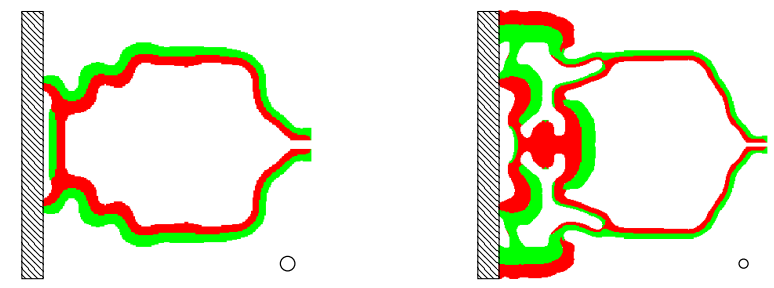

Figure 6. Polarization profile for $k_{\text {out }}=20 \mathrm{~N} / \mathrm{m}$ and $L=3000 \mu \mathrm{m}$ (left) and $L=8000 \mu \mathrm{m}$ (right).

with one single piezoelectric film on top of the structure.

From now on, for the sake of simplicity, only the polarization profile of the blue print will be shown, since this projection contains all the information about the topology of the structure. Figure 5(left) shows an optimized design for $k_{\text {out }}=100 \mathrm{~N} / \mathrm{m}$ and figure 5 (right) for $k_{\text {out }}=200 \mathrm{~N} / \mathrm{m}$. The displacements in these cases are $1.66 \mu \mathrm{m}$ and $1.34 \mu \mathrm{m}$, respectively. The increase of $k_{\text {out }}$ implies an increase of the stiffness of the structure, which in turns reduces the output displacement.

In figure 6 are shown two example with different size of domain $\Omega$. The length is fixed to $L=3000 \mu \mathrm{m}$ and the mesh to $240 \times 240$ elements in figure 6(left). These two parameter are set to $L=8000 \mu \mathrm{m}$ and $320 \times 320$ elements in figure 6(right). The output displacements for these cases are $1.24 \mu \mathrm{m}$ in the first case and $5.00 \mu \mathrm{m}$ in the second one. It is easy to check that the larger the size, the larger the displacement, since the area covered by electrode is bigger.
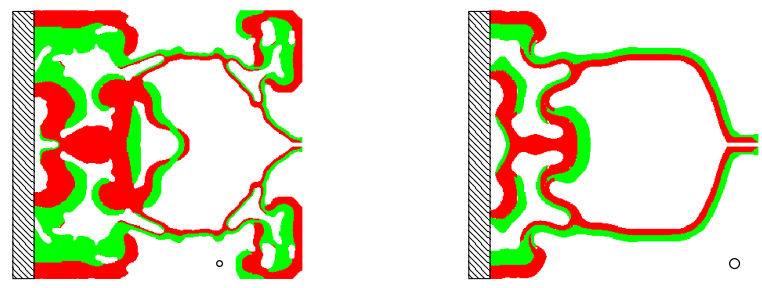

Figure 7. Polarization profile for $k_{\text {out }}=20 \mathrm{~N} / \mathrm{m}, L=8000 \mu \mathrm{m}$ and minimum length size of $160 \mu \mathrm{m}$ (left) and $340 \mu \mathrm{m}$ (right).

Finally, figure 7 shows the effect of varying the minimum length scale for $L=8000 \mu \mathrm{m}$. The feature size is fixed to $160 \mu \mathrm{m}$ in figure 7 (left) and to $340 \mu \mathrm{m}$ in figure 7 (right). The displacement at the output port is $6.09 \mu \mathrm{m}$ and $4.46 \mu \mathrm{m}$, respectively. The decrease in the minimum length scale implies the appearance of smaller parts in the structure. Somehow the minimum length scale can be understood as a constraint in the problem, in the sense that a bigger length scale imposed implies a reduction of the displacement at the output port. 


\section{CONCLUSIONS}

In this work a systematic procedure to design piezoelectrically actuated microgrippers is presented. The objective is the maximization of the displacement at the output port, while the out-of-plane bending at some points placed at the jaws is suppressed. The latter is the main novelty introduced. This suppression must be taken into account in the design, otherwise the out-of-plane bending produced by the unsymmetrical lamination jeopardizes the functionality of the gripper. From an optimization point of view is not possible to suppress this bending in the whole domain, this is the reason for suppressing only at some points of the jaws.

Additionally, a robust approach is used with two goals. The first one is controlling the minimum length scale in the whole domain, avoiding the appearance of hinges (thin areas that are prone to break due to the high stress generated there). The second goal is the minimization of the target (displacement at the output port) to small manufacturing errors. Fabrication techniques at microscale are very complicated and hence optimized designs should be insensitive to e.g. under- and over-etching.

Several grippers have been designed varying parameters such as the length of the domain $\Omega$, the stiffness $k_{\text {out }}$ of the output spring and the minimum length scale imposed. The method proposed has shown quite good performance suppressing the out-of-plane bending while the displacement at the output port is maximized, which was the objective of this work.

\section{ACKNOWLEDGMENTS}

The work of J.C. Bellido and A. Donoso has been funded by MINECO (Spain) through grant MTM2013-47053$\mathrm{P}$ and Junta de Comunidades de Castilla-La Mancha (Spain) through grant PEII-2014-010-P. The work of O. Sigmund has been supported by the research project Sapere Aude TOpTEn (Topology Optimization of Thermal ENergy systems) from the Danish Council for Independent Research, grant: DFF-4005-00320. The work of J.L. Sánchez-Rojas was supported by Spanish MINECO project TEC2015-67470-P and regional JCCM project PEIC-2014-001-P.

\section{REFERENCES}

[1] Sigmund, O., "On the design of compliant mechanisms using topology optimization," Mech Struct Mach 25(4), 493-524 (1997).

[2] Jonsmann, J., Sigmund, O., and Bouwstra, S., "Compliant thermal microactuators," Sens Act A Phys 76(13), $463-469$ (1999).

[3] Jensen, J. and Sigmund, O., "Topology optimization for nano-photonics," Laser Photonics Rev 5(2), 308-321 (2011).

[4] Sigmund, O. and Torquato, S., "Design of materials with extreme thermal expansion using a three-phase topology optimization method," J Mech Phys Solids 45(6), 1037 - 1067 (1997).

[5] Pedersen, N. L., "Maximization of eigenvalues using topology optimization," Struct Multidisc Optim 20, 2-11 (2000).

[6] Sigmund, O., Torquato, S., and Aksay, I. A., "On the design of 1-3 piezo-composites using topology optimization," J Mater Res 13(4), 1038-1048 (1998).

[7] Silva, E. C. N. and Kikuchi, N., "Design of piezoelectric transducers using topology optimization," Smart Mater Struct 8(3), 350-364 (1999).

[8] Kögl, M. and Silva, E. C. N., "Topology optimization of smart structures: design of piezoelectric plate and shell actuators," Smart Mater Struct 14(2), 387-399 (2005).

[9] Carbonari, R. C., Silva, E. C. N., and Nishiwaki, S., "Optimum placement of piezoelectric material in piezoactuator design," Smart Mater Struct 16(1), 207-220 (2007).

[10] Luo, Z., Gao, W., and Song, C., "Design of multi-phase piezoelectric actuators," J Intell Mater Syst Struct 21(18), 1851-1865 (2010).

[11] Kang, Z. and Tong, L., "Integrated optimization of material layout and control voltage for piezoelectric laminated plates," J Intell Mater Syst Struct 19, 889-904 (2008). 
[12] Kang, Z. and Tong, L., "Topology optimization-based distribution design of actuation voltage in static shape control of plates," Comput Struct 86(19-20), 1885-1893 (2008).

[13] Kang, Z., Wang, R., and Tong, L., "Combined optimization of bi-material structural layout and voltage distribution for in-plane piezoelectric actuation," Comput Methods Appl Mech Eng 200(13-16), 1467-1478 (2011).

[14] Kang, Z., Wang, X., and Luo, Z., "Topology optimization for static shape control of piezoelectric plates with penalization on intermediate actuation voltage," J Mech Des 134(5), 051006 (2012).

[15] Ruiz, D., Bellido, J. C., Donoso, A., and Sánchez-Rojas, J. L., "Design of in-plane piezoelectric sensors for static response by simultaneously optimizing the host structure and the electrode profile," Struct Multidisc Optim 48(5), 1023-1026 (2013).

[16] Ruiz, D., Bellido, J. C., and Donoso, A., "Design of piezoelectric modal filters by simultaneously optimizing the structure layout and the electrode profile," Struct Multidisc Optim 53, 715 - 730 (2016).

[17] Kucera, M., Wistrela, E., Pfusterschmied, G., Ruiz-Díez, V., Manzaneque, T., Hernando-García, J., Sánchez-Rojas, J. L., Jachimowicz, A., Schalko, J., Bittner, A., and Schmid, U., "Design-dependent performance of self-actuated and self-sensing piezoelectric-aln cantilevers in liquid media oscillating in the fundamental in-plane bending mode," Sens Act B Chem 200, 235 - 244 (2014).

[18] Wang, F., Lazarov, B. S., and Sigmund, O., "On projection methods, convergence and robust formulations in topology optimization," Struct Multidisc Optim 43(6), 767-784 (2011).

[19] Bendsøe, M. P. and Sigmund, O., "Material interpolation schemes in topology optimization," Arch Appl Mech 69(9-10), 635-654 (1999).

[20] Bendsøe, M. P. and Sigmund, O., [Topology optimization: theory, methods and applications], 2nd Edition, Springer (2003).

[21] Oñate, E., [Cálculo de Estructuras por el Método de Elementos Finitos, Análisis estático lineal], 2nd Edition, CIMNE (1995).

[22] Moheimani, S. O. and Fleming, A. J., [Piezoelectric Transducers for Vibration Control and Damping], 2st Edition, Springer (2006).

[23] Gibbs, G. P. and Fuller, C. R., "Excitation of thin beams using asymmetric piezoelectric actuators," $J$ Acoust Soc Am 92(6), 3221-3227 (1992).

[24] Bourdin, B., "Filters in topology optimization," Int J Numer Meth Eng 50(9), 2143-2158 (2001).

[25] Guest, J. K., Prvost, J. H., and Belytschko, T., "Achieving minimum length scale in topology optimization using nodal design variables and projection functions," Int J Numer Meth Eng 61(2), 238-254 (2004).

[26] Bruns, T. E. and Tortorelli, D. A., "Topology optimization of non-linear elastic structures and compliant mechanisms," Comput Methods Appl Mech Eng 190(26-27), 3443-3459 (2001).

[27] Svanberg, K., "The method of moving asymptotes-a new method for structural optimization," Int J Numer Meth Eng 24(2), 359-373 (1987). 